\title{
AOR
}

Selected Papers of \#AolR2021:

The 22nd Annual Conference of the

Association of Internet Researchers

Virtual Event / 13-16 Oct 2021

\section{ANALYZING PLATFORM POWER IN THE CULTURAL INDUSTRIES}

\author{
David B. Nieborg \\ University of Toronto \\ Thomas Poell \\ University of Amsterdam \\ Brooke Erin Duffy \\ Cornell University
}

\section{Introduction}

Across cultures and contexts, digital platforms like YouTube, TikTok/Douyin, WeChat, and Spotify are fundamentally reshaping both the processes and products of cultural production-from music and news to entertainment and advertising (Poell et al., 2021). But, despite considerable attention to the perverse power of algorithms in various spheres of social and economic life, we contend that existing political economic frameworks fail to account for the distinctiveness of the cultural industries. These industries are, as sociologist Gina Neff (2012) contends, sites where value is "communicatively constituted and mediated" as part of the marketplace's increasing reliance on the production of "symbolic, informational, and aesthetic goods" (p. 29).

To help systematize analysis of the relations and flows of power between platforms and cultural producers, this paper introduces a framework that is carefully attuned to the historical and industrial specificities of cultural production. The framework makes clear that, while the relationship between platforms and cultural producers is staggeringly uneven and, at times, highly volatile, it should be understood as one of mutual dependence. That is, platforms exert mechanisms of power over the phases of the creation, distribution, monetization, and marketing of culture; but they also furnish space for negotiation and contestation. Acknowledging this requires a framework that is less deterministic and sensitive to the nuance inherent in cultural production.

\section{Towards a nuanced understanding of platform power}

Key to understanding the reconfigured power relations in the cultural industries is the recognition that cultural producers are increasingly becoming institutional actors in multi-sided markets. While legacy media industries were predominantly sustained by 
single or dual (i.e., advertising and audience) revenue streams, platform companies in the cultural sector-e.g., Facebook, Google, Tencent, and others-not only aim to acquire end-users and advertisers, but also complementors. The latter group consists of individual entrepreneurs and companies that 'complement' the products and services provided by the platform (i.e., a wide variety of cultural producers, data intermediaries, and others). To attract and retain these complementors, platform companies continuously adapt pricing structures, revenue models, developer agreements, moderation practices, algorithmic filtering mechanisms, and so on. Throughout such processes, platforms try to strike a balance between openness and control of their platform boundaries. This, in turn, compels cultural producers to adopt and integrate decisions, often under highly precarious circumstances.

To be sure, the platform-wrought pressures facing cultural producers are not without precedent. To the contrary, cultural producers have long negotiated control with gatekeepers, intermediaries, and immensely powerful transnational media conglomerates (Mirrlees, 2013). But, with cultural producers becoming increasingly platform-dependent, it seems critical to conceptually delineate their spaces for negotiation and strategic decision-making. As such, this paper offers a conceptual blueprint for analyzing three key variables that inform a more systematic analysis of platform-dependent cultural production.

\section{Variables}

Building on research in science and technology studies (STS), software studies, political economy, business studies, and media industries studies, the paper presents a new analytical framework to analyse the evolving power relationship between platforms and cultural producers. We argue that the decision space of cultural producers as platform complementors is shaped by three key variables: 1) platform evolution, 2) cultural industry segments, and 3) stages of production.

The first variable impacting the bargaining power of complementors is "platform evolution," which describes the stage of a platform's infrastructural development, its (in)ability to generate revenue, and its rate of adoption and subsequent uptake by endusers. STS scholarship reminds us that there is no set trajectory or predetermined path along which (media) technologies evolve. Instead, as José van Dijck argues (2013), platforms go through a marked phase of "platform ambiguity" and "interpretative flexibility," during which it is anything but ensured what a platform is meant to do, who a platform opens its boundaries to, and how its business model will develop. Put differently, digital infrastructures-and we consider the platform services operated by Alibaba, Amazon, Apple and other platforms to be prime examples of such-are inherently contingent and unpredictable; platform infrastructures (i.e., Application Programming Interfaces, tools, documentation, etc.) are always evolving based on endusers' datafied feedback, a platform's business model, or tweaks to its governance framework (Poell et al., 2021).

Consequently, platform evolution impacts complementors' economic opportunities in variable ways. For instance, platform companies tend to provide cultural producers with more favorable economic conditions early in their lifecycle (Rietveld et al., 2020). Vice versa, those conditions tend to change when a platform 'matures', i.e., when it reaches 
a certain adoption threshold. For cultural producers, these stages affect the space of negotiations vis-à-vis platforms.

Second, platform adoption among cultural producers differs considerably among industry segments. Historically, some parts of the cultural industries-games and social media entertainment-have been platform-dependent, whereas other segments-news and music-have histories far predating platforms. The latter set of industry segments tend to have more options to operate independently from platforms. This diversity is in part due to the strategic choices of cultural producers, but it also owes much to the 'nature' of specific modes of cultural production, including the historical trajectories of particular industry segments in particular cultural contexts (Miège, 2011). Thus, cultural producers becoming platform-dependent by no means signals an all-encompassing logic; nor does it affect all industries equally. As the relation between cultural producers and platforms is highly variable, the space of negotiations and strategic decision-making for these producers also widely varies.

This second variable impacts a third variable: the stage of production. Depending on whether one focuses on cultural creation, distribution, marketing, or monetization, cultural producers have more or less autonomy to operate independently from platforms in an economically sustainable fashion. Providing access to large and diverse pools of end-users, platforms have become especially indispensable in the distribution phase. For example, the distribution of apps, streaming content, or social media entertainment all involve platform-dependent distribution. Conversely, during the stages of cultural creation, marketing, and monetization, the supposed dominant role of platforms is less straightforward and not inherently platform-dependent, and therefore leaves cultural producers with other options.

\section{Conclusion}

Challenging essentialist theories of platform dominance, this paper argues that claims of platform power need to be qualified in the context of industry- and culture-specific inquiries. The analysis we introduced testifies to both the power of platforms and the spaces of negotiations and decision-making among cultural producers. To understand how cultural producers depend on platforms and how changes in platform markets, infrastructures, and governance affect their operations, we need to carefully analyse how platforms and cultural producers interact in specific settings. And, by systematizing the research-exploring the variation in platform evolution, industry segments, and stages of cultural production-we gain a comprehensive understanding of how platform power takes shape in the contemporary cultural industries.

\section{References}

Miège, B. (2011). Theorizing the cultural industries: Persistent specificities and reconsiderations. In J. Wasko, G. Murdock, \& H. Sousa (Eds.), The handbook of political economy of communications (pp. 83-108). Blackwell.

Mirrlees, T. (2013). Global entertainment media: Between cultural imperialism and cultural globalization. Routledge. 
Neff, G. (2012). Venture labor: Work and the burden of risk in innovative industries. MIT press.

Poell, T., Nieborg, D. B., \& Duffy, B. E. (2021). Platforms and Cultural Production. Polity

Rietveld, J., Ploog, J. N., \& Nieborg, D. B. (2020). The coevolution of platform dominance and governance strategies: Effects on complementor performance outcomes. Academy of Management Discoveries, 6(3), 488-513.

van Dijck, J. (2013). The culture of connectivity: A critical history of social media. Oxford University Press. 